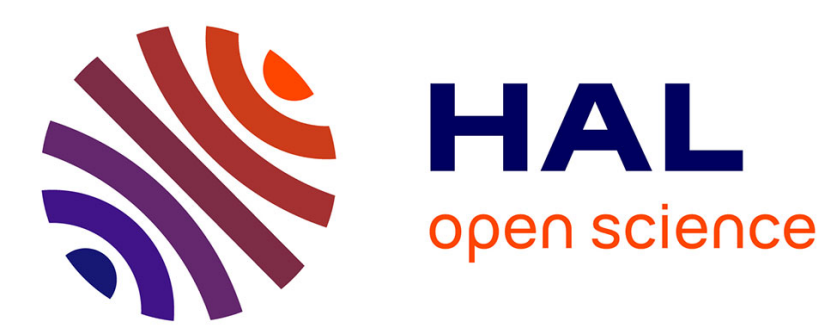

\title{
In situ experiments and seismic analysis of existing buildings. Part II: Seismic integrity threshold
}

\author{
Claude Boutin, Stéphane Hans, Erdin Ibraim, Pierre Roussillon
}

\section{To cite this version:}

Claude Boutin, Stéphane Hans, Erdin Ibraim, Pierre Roussillon. In situ experiments and seismic analysis of existing buildings. Part II: Seismic integrity threshold. EARTHQUAKE ENGINEERING \& STRUCTURAL DYNAMICS, 2005, 34 (12), pp.1531-1546. hal-00941482

\section{HAL Id: hal-00941482 \\ https://hal.science/hal-00941482}

Submitted on 14 Feb 2014

HAL is a multi-disciplinary open access archive for the deposit and dissemination of scientific research documents, whether they are published or not. The documents may come from teaching and research institutions in France or abroad, or from public or private research centers.
L'archive ouverte pluridisciplinaire HAL, est destinée au dépôt et à la diffusion de documents scientifiques de niveau recherche, publiés ou non, émanant des établissements d'enseignement et de recherche français ou étrangers, des laboratoires publics ou privés. 


\title{
In situ experiments and seismic analysis of existing buildings. Part II: Seismic integrity threshold
}

\author{
C. Boutin ${ }^{1, *, \dagger}$, S. Hans ${ }^{1}$, E. Ibraim ${ }^{2}$ and P. Roussillon ${ }^{1}$ \\ ${ }^{1}$ Laboratoire Geomateriaux, Departement Genie Civil et Batiment, URA CNRS 1652, Ecole Nationale des \\ Travaux Publics de l'Etat, rue Maurice Audin, 69518 Vaulx-en-Velin Cedex, France \\ ${ }^{2}$ Department of Civil Engineering, Queen's Building, University of Bristol, University Walk, Bristol \\ BS8 1TR, U.K
}

\begin{abstract}
SUMMARY
The interest of in situ measurements (presented in Part I paper) for a seismic assessment of existing buildings is analysed in this paper. It is shown that the experimental modal characteristics obtained on regular concrete structures are described successfully by suited Timoshenko beam modelling. For a given structure, taking into account the experimental data, the corresponding beam model, and choosing the maximum tensile strain of concrete as damage criterion for key structural elements, a maximum level of the ground acceleration can be determined. This so-called seismic integrity threshold is directly related to the onset of structural damages. This new approach is illustrated on one of the studied buildings. The advantages of using ambient vibrations survey for the vulnerability assessment of existing buildings are discussed.
\end{abstract}

KEY WORDS: in situ test monitoring; existing buildings; ambient vibrations; Timoshenko beam; seismic integrity threshold

\section{INTRODUCTION}

Different scales require different methodologies to establish a seismic diagnosis. If a single strategic building is concerned, a fine detailed analysis can be performed for an accurate assessment. If a whole city is concerned, the overall seismic vulnerability can be addressed using statistical approaches such as that initiated by Benedetti et al. [1]. At an intermediate scale, the question may be focused on a class of particular structures such as hospitals, schools, fire stations, but also on ordinary or industrial structures. The statistic method becomes then

*Correspondence to: C. Boutin, Laboratoire Geomateriaux, Departement Genie Civil et Batiment, URA CNRS 1652, Ecole Nationale des Travaux Publics de 1'Etat, rue Maurice Audin, 69518 Vaulx-en-Velin Cedex, France.

†E-mail: claude.boutin@entpe.fr

Contract/grant sponsor: French Ministry of Environment

Contract/grant sponsor: French Association of Earthquake Engineering 
non-efficient, and the use of sophisticated methods for a large number of buildings would be very time-consuming at a prohibitive cost. In this situation, preliminary diagnosis devoted to specific structures such as that proposed by Fritz de la Orta [2] have to be improved. This study, developed from the analysis of a few real buildings, proposes a methodology as simple and reliable as possible adapted to such a diagnosis level. This approach leads to define a practical criterion of interest for vulnerability diagnosis, built from both experimental data and realistic, tough simple, building modelling.

In the first paper [3], it was shown that dynamic monitoring tests as ambient vibration measurements conveniently provide valuable information on existing buildings that are usually poorly documented [4]. To go further, a link has to be established between experimental data and a seismic diagnosis.

In the present paper, this purpose is achieved in two steps. The first step consists in relating the modal characteristics extracted from the measurements to the mechanical functioning of the structure. This is developed in the first section, where it is shown that shear beam and more generally, Timoshenko beam describes the dynamic behaviour of the tested buildings with a good accuracy. In the second step, presented in the next section, these results are used to deduce the internal strains associated to the modal deformations. Thus, using the maximum tensile strain of concrete as damage criterion for the key structural elements, a seismic integrity threshold (SIT), suited to a given structure, is derived. This normalized acceleration amplitude (according to the seismic codes) is directly related to the onset of structural damages. This new approach is illustrated by using the in situ records of one of the studied buildings. This study case shows that the SIT value and the gap between the SIT and the acceleration level required by the codes are of interest for a first seismic analysis. Finally, the advantages of coupling simple modelling and ambient vibrations survey for the seismic diagnosis of existing buildings are discussed.

Note: The data concerning the two tested buildings $\mathrm{C}$ and $\mathrm{G}$ and their modal characteristics are not reproduced in the present paper. The reader may refer to the Part I paper where they are presented in detail.

\section{RELEVANT BEAM MODEL}

It was established, in Part I paper, that ambient vibration measurements can provide the modal characteristics of a building with a good reliability in the whole quasi-elastic domain (despite the presence of slight perturbations induced by a few 'parasite' effects). Moreover, experiments on modified buildings indicate that this data are directly linked with the response of the key structural elements. However, the link between the modal deformation at the global building scale, and the internal strain at the structural element scale-where the damages may occur-is not explicitly given by the experimental data.

The aim of this section is to fill this gap by determining relevant, though simple, beam modelling whose validity could be easily checked with experimental data. The analysis is here focused on regular concrete buildings made of a sufficient number of identical storey (say more than five). It is shown that the experimental quasi-elastic behaviour of such structures (more precisely the fixed base behaviour (SB), see Part I) can be reduced to the behaviour of a vertical continuous cantilever beam. In addition, in order to have a clear mechanical understanding at both global and local scales, the beam model integrates in a very synthetic way the essential parameters of the dynamic behaviour. 


\subsection{The Timoshenko beam model considered}

Among many possible beam modelling (see Reference [5]), we consider a particular description that may include the main mechanisms occurring in a regular building. The behaviour of a regular structure of height $H$ made of elastic material (of Young's modulus $E$ ) is governed by the spatial distribution of columns, bearing walls and floors diaphragms constituting the storey. Considering vibrations in a horizontal main direction, the deformations result from two contributions (see for instance Reference [6]):

(i) the bending motion characterized by the bending parameter EI,

(ii) the shear motion characterized by the shear parameter $K$.

Denoting by $\Lambda$ the linear mass of a storey and neglecting the rotation inertia, these basic assumptions lead to a specific Timoshenko beam. The main features are briefly given hereafter (see Appendix A for more details).

In harmonic regime, the horizontal translation motion of the section, $U(x, t)=U(x) \mathrm{e}^{\mathrm{i} \omega t}$, where $x$ is the position along the beam, $\omega$ the angular frequency and $t$ the time, is governed by

$$
\mathrm{EI} U^{(4)}(x)+\frac{\mathrm{EI}}{K} \Lambda \omega^{2} U^{(2)}(x)=\Lambda \omega^{2} U(x)
$$

The dimensionless parameter $C=\mathrm{EI} / K L^{2}$, where $L=H / 2 \pi$, characterizes the nature of the Timoshenko beam. This model beam degenerates into an usual Euler-Bernoulli beam when $C=0$, and into a pure shear beam when $C=+\infty$. The usual modal analysis applied to this Timoshenko beam clamped at the base and free at the top, gives the $k$ th eigenfrequency

$$
f_{k}=\frac{1}{2 \pi L} \frac{\delta_{1 k}^{2}}{\sqrt{\left(\Lambda L^{2} / \mathrm{EI}\right)+\delta_{1 k}^{2} \Lambda / K}} \quad \text { with } \delta_{1 k} \simeq 2 k+1 ;(k \geqslant 2)
$$

The distribution of the eigenfrequencies strongly depends on the nature of beam: the sequence is almost homothetic to the odd integer sequence for beam where the shear dominates (say $C>5$ ), whereas, for beams where the bending dominates (say $C<0.05$ ), the sequence is almost homothetic to the square odd integer sequence $(2 k+1)^{2}$. Moreover, the ratio between two eigenfrequencies depends on the parameter $C$ only. For instance, as illustrated in Figure 1, the ratio of the two first frequencies monotonically decreases when $C$ increases. Therefore, there is a biunivoque relationship between this ratio and the beam parameter $C$.

It is worth mentioning that this observation provides a very simple way to identify the nature of the beam from the sequence of the measured eigenfrequencies or even from the $f_{2} / f_{1}$ ratio.

\subsection{Simple a priori assessment of the Timoshenko beam parameters}

To go further in the possible identification of the building with a beam, we have now to specify how the shear $(K)$ and bending $(E I)$ stiffness and linear density $(\Lambda)$ can be determined.

Assessments of the three needed parameters can be derived from some basic information on the structure (see Part I paper) and reasonable assumptions on the constitutive material and internal deformations. 


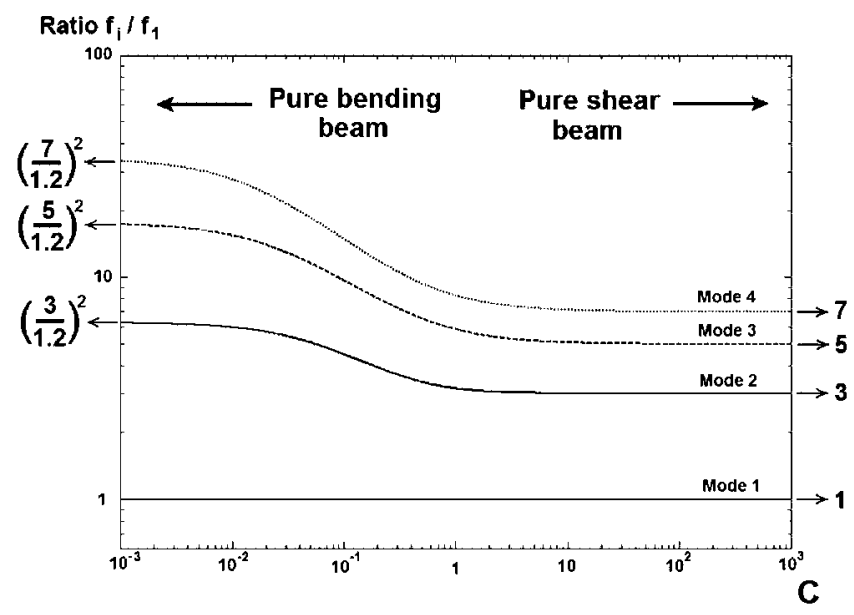

Figure 1. Evolution of frequencies ratio $f_{i} / f_{1}$ in function of parameter $C$.

The linear density $\Lambda$ is simply estimated with a good accuracy from the geometrical characteristics given by the drawings, and the usual density of materials (a mean value of $2.3 \mathrm{t} / \mathrm{m}^{3}$ is adopted for the reinforced concrete).

The elastic beam constants are estimated using the following assumptions:

(i) perfectly rigid connections are assumed between the structural elements;

(ii) the elastic modulus of the material is assumed to have an usual value (a modulus $E$ of $20 \mathrm{GPa}$ and Poisson ratio $v=0.2$ is taken for the reinforced concrete);

(iii) the floors are assumed infinitely rigid (this assumption is only partially justified and considered in a first level of analysis).

The storey shear stiffness (in a given main horizontal direction) is derived by imposing between two successive rigid floors a differential horizontal drift. The same inter-storey drift applies to the columns and bearing walls clamped on the floors at their two extremities. According to their stiffness in this direction, the global horizontal force is obtained by summing up the contributions of all the structural elements. The coefficient relating the global force to the shear strain (i.e. the differential drift divided by the height of the storey) defines the global shear stiffness in the considered direction. In other words, the global shear stiffness is obtained by adding the rigidities of the elements divided by the height of the storey. Without going into details, the elements stiffness is given by usual strength of materials static formulae that include bending stiffness (dominating for columns and out of plane wall motions) and shear stiffness (dominating for in-plane wall motions).

The storey bending stiffness (in a given main horizontal direction) is obtained in a similar way. For two successive rigid storeys, a differential rotation around a given horizontal axis will lead a linear distribution of positive and negative vertical tensile strains in columns and bearing walls. According to their normal stiffness, a distribution of traction and compression forces will result and a global momentum will be generated (the position of rotation axis corresponds to a zero global compressive force). The coefficient relating the momentum to the curvature (i.e. the differential rotation divided by the high $h$ of the storey) defines the global bending stiffness in the considered direction. 
Table I. Results of direct estimation on tested buildings.

\begin{tabular}{lccccc}
\hline Building & $\mathrm{C}$ & & \multicolumn{2}{c}{$\mathrm{G}$} \\
\cline { 1 - 2 } \cline { 5 - 6 } Direction & Longitudinal & & Longitudinal & Transversal \\
\hline Linear mass $(t / m)$ & 114 & & & 110 & 115600 \\
Shear parameter K $(\mathrm{MN})$ & 2140 & & 27830 & & 354 \\
Bending inertia I $\left(m^{4}\right)$ & 19.7 & & 1.79 & 0.08 \\
Parameter $C$ & $3.63-11.5-17.8$ & & $2.58-7.91-14.12$ & $2.24-10.54-23.07$ \\
Estimated frequencies $\left(f_{1} ; f_{2} ; f_{3}\right)$ & $4.45-14.1-23.5$ & & $2.15-7.24-13.97$ & $1.56-6.64-14.0$ \\
with $E=20$ GPa & &
\end{tabular}

Table I gives the numerical results obtained for building $C$ (in the longitudinal direction) and building $\mathrm{G}$ in both longitudinal and transversal directions. Clearly the value of parameter $C \approx 20$ indicates a dominating shear beam behaviour for building $\mathrm{C}$ in the longitudinal direction, and a Timoshenko beam behaviour in both directions for building $\mathrm{G}(0.1<C<2)$. The first three frequencies calculated from these estimations are comparable with the experimental values (at least for these modes), meaning that this simple approach can provide a reasonably good description of the building behaviour.

\subsection{Experimental estimate of the beam parameters}

To complement the previous assessments, the beam parameters can also be estimated independently from the experimental sequence of eigenfrequencies.

2.3.1. Building $C$. According to the previous result, building $\mathrm{C}$ can simply be modelled, in its longitudinal direction, by a pure shear beam characterized by its shear stiffness $K=E * S_{\mathrm{s}}$, where $S_{\mathrm{s}}$ is a purely geometric quantity associated to the structure (the Poisson's coefficient is supposed known). Then, considering the estimated linear density and the geometry as given data, the identification of the beam parameter reduces to the estimation of the last unknown, i.e. the Young's modulus. It is therefore possible to adjust the modulus of the material to fit the first eigenfrequency of the model with the experimental value $(4.45 \mathrm{~Hz})$. In the present case, the model is fitted when taking a Young's modulus equal to $31 \mathrm{GPa}$. Moreover, using this realistic value, the first three model eigenfrequencies are, respectively, 4.45, 13.3 and $21.8 \mathrm{~Hz}$ very close to the experimental ones: $4.45,14.1$ and $23.5 \mathrm{~Hz}$.

2.3.2. Building $G$. In the two main directions of building $\mathrm{G}$, two Timoshenko beam models were found to describe the dynamic behaviour. In this case, a two parameters problem has to be solved, with for unknowns the parameter $C$ and for instance the parameter $K$ linked to the shear stiffness. As mentioned above, the ratio of the two first eigenfrequencies $f_{2} / f_{1}$ corresponds to a unique value of parameter $C$, and consequently by using the experimental ratio $f_{2} / f_{1}$, a experimental value of $C$ is determined.

Then, considering here again the estimated linear density and the geometry as given data, it is possible to fit the first experimental frequency by adjusting the Young's modulus of the 
Table II. Experimental refitting-building G.

\begin{tabular}{lccc}
\hline Building & \multicolumn{2}{c}{$\mathrm{G}$} \\
Direction & Longitudinal & Transversal \\
\hline Experimental frequencies $(\mathrm{Hz})$ & $2.15-7.24-13.97-20.5$ & $1.56-6.64-14.0$ \\
Experimental ratio $f_{2} / f_{1}$ & 3.37 & 4.26 \\
Experimental $C$ & 0.50 & 0.13 \\
Experimental Young's modulus $(\mathrm{GPa})$ & 21 & 21 \\
Fitted beam model frequencies $(\mathrm{Hz})$ & $2.15-7.24-13.96-20.1$ & $1.56-6.64-14.0$ \\
\hline
\end{tabular}

material. The fitting process performed independently for both directions leads to an identical modulus value of $21 \mathrm{GPa}$.

Table II gives the results obtained by this procedure. The comparison with Table I shows that the fitting of frequency ratio improves the rough a priori estimate of parameter $C$ for building $G$.

\subsection{Checking the relevancy of the continuous beam modelling}

Having the beam parameters calibrated by the in situ data, the reliability of the modelling can be checked by complementary comparisons. For this purpose, the mode shapes and eigenfrequencies of higher modes are calculated and compared to the experimental data.

It is interesting to note that the succession of eigenfrequencies is well described by the beam model (see Table II), up to the third frequency for building $\mathrm{C}$-with only the first frequency fitted - and up to the fourth for building G, with only the two first frequencies fitted.

Figures 2 and 3 present the comparison of the experimental and theoretical mode shapes for the three cases studied. It is worth mentioning that, despite the fact that the modal shapes were not involved in the fitting process, there is a very good agreement between experiments and modelling, even for the modes associated to non-fitted frequencies. It is also worth noting that the first mode curvature is consistent with the beam modelling: positive for shear beam, as building $\mathrm{C}$; with an inflexion for Timoshenko beam, as building $\mathrm{G}$ in longitudinal direction; negative for beam with dominating bending effect, as building $\mathrm{G}$ in transversal direction.

Let us also mention that the Young's modulus values determined (between 20 and $30 \mathrm{GPa}$ ) are realistic and seem to support the reliability of this approach. Finally, the consistency of these results obtained for three different cases leads to think that even moderately tall buildings can actually be considered as beams, whose parameters can be determined rather simply from basic information and in situ measurements.

\section{INTEGRITY THRESHOLD}

The question addressed here is: how could these in situ experimental data, consistently described by a simple beam modelling suited to the structure, contribute to a seismic diagnosis?

Since a reliable description of the (quasi) elastic building behaviour (including all the mechanically active elements) is available, the idea consists in determining the limit of this elastic domain. More precisely, it is intended to estimate the seismic acceleration 


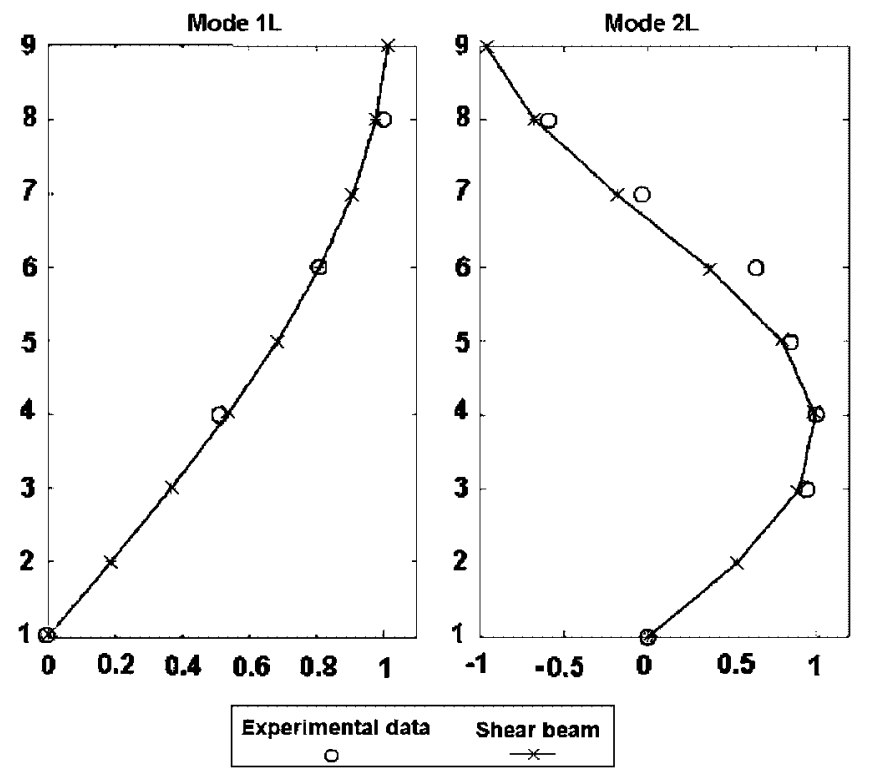

Figure 2. Modelling-experiment comparison of modal shapes for the building C (Longitudinal direction).

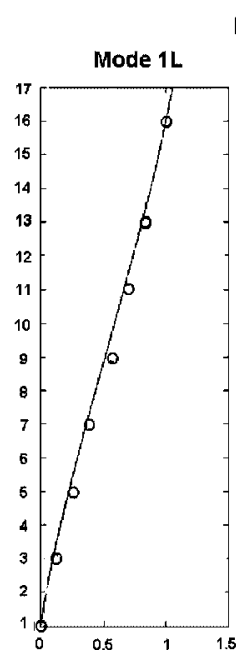

Longitudinal direction
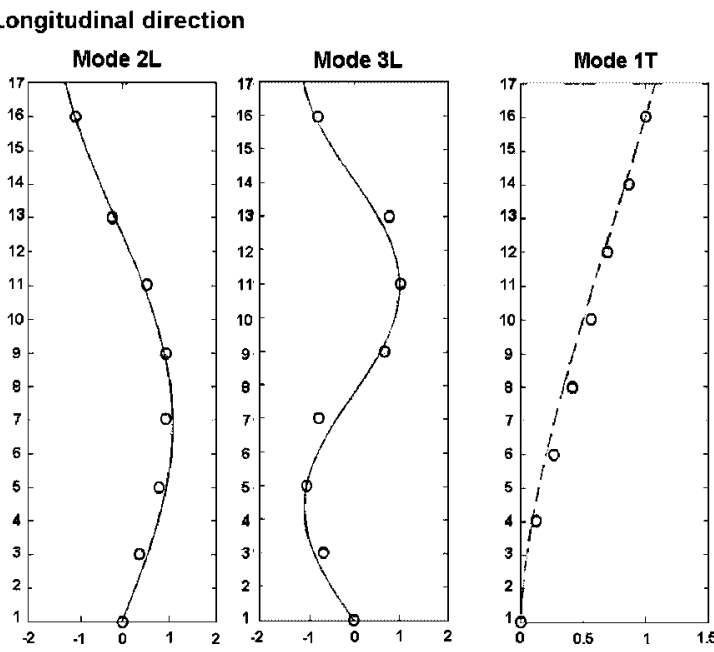

Transversal direction

Experimental data

Timoshenko beam

o $\quad-c_{L}=0.5---c_{T}=0.13$

Figure 3. Modelling-experiment comparison of modal shapes for the building G.

level - related to the normalized earthquake spectra given by the code-which generates the onset of first structural damages. Below this level, the so-called SIT, the structure remains elastic, i.e. undamaged. 
In the following, the principle of the SIT's determination is described and an application to a case study is presented.

\subsection{Principle}

3.1.1. Criterion for the onset of the structural damage. A key issue in this approach is to define the criterion for the onset of structural damage. It should be emphasized that in this paper, the discussion is especially focused on reinforced concrete structures. The actual amount of steel reinforcements (even if low as in the studied buildings) and their exact distribution in the sections are unknown for most of existing structures. Therefore, it would not be relevant to adopt a stress criterion for reinforced concrete-expressed in maximum compressive or tensile stress - since such a criterion would include the effect of reinforcements. To overcome this difficulty, we use the well known fact that, independently of the presence of reinforcements, the concrete matrix cannot sustain tensile strains greater than a limit of $10^{-4}(\mathrm{~m} / \mathrm{m}$ ) (for usual concrete).

Subsequently, this simple criterion of maximum concrete tensile strain is adopted. Below this limit, the concrete (and thus the reinforced concrete) remains intact; and above this limit, the cracking of the concrete begins and weakens the reinforced concrete elements. Usually, at this first level of damage, the unconfined concrete reinforcement cover is ejected, thus reducing the effective section and inertia of the elements.

Let us underline that the choice of a strain criterion is consistent with the displacementbased methods [7], and with the concept of maximum storey drift [8].

\subsubsection{Method. The SIT value is deducted from:}

(i) the quasi-elastic behaviour-within the seismic frequency range-identified from ambient vibration tests,

(ii) the beam model deducted from experiments and straightforwardly related to the internal building structure,

(iii) the damage criterion.

It is clear that, if the structure was purely elastic, its temporal response to any seismic excitation could be fully determined. Obviously, this response will coincide with that of the real structure until the damage criterion is reached (at this moment, 'elastic' and 'damaged' responses diverge). When focusing on seismic signal corresponding to normalized earthquake spectra, the level of excitation is simply characterized by the normalized acceleration. If we consider several seismic signals with increasing normalized accelerations, the quasi-elastic response of the building increases quasi-linearly with the excitation amplitude. This will be true until a first damage appears in some part of the structure. At this moment, the corresponding normalized acceleration will be called the SIT. It is interesting to note that, in this method, the localization of the first structural damages can be detected from the deformed structural shape defined according to its quasi-elastic vibration modes.

3.1.3. SIT calculation and first mode approximation. The calculation of the SIT could be performed through common linear structural dynamic numerical methods, the model being fitted with the experimental data. However, in order to give a better insight of the method, all the process will be illustrated in Section 3.2 using building $\mathrm{C}$ case study where the 
calculations can be developed almost analytically taking only into account the first mode. This approximation - done only for the sake of simplicity - consists in considering that the first mode is mainly responsible for the structural deformations. This rough simplification can be partly justified:

- the frequency distribution of seismic energy is such that for usual buildings with well separated eigenfrequencies (for instance 4.45 and $14.1 \mathrm{~Hz}$ for building $\mathrm{C}$ ), the maximum of energy is concentrated on the first mode,

- in addition, the participation factor, and then the effective amplitude, are smaller for higher modes.

It is then possible to estimate the amplitude of the first mode displacement which would lead to the onset of the structural damages. Using the normalized elastic response spectra provided by the codes, this modal amplitude can be converted into the corresponding acceleration level, i.e. the SIT.

3.1.4. Practical interest of the SIT value. The SIT corresponds to a seismic elastic limit, whereas the safety strategy against earthquake is based on ductility. In these conditions, it is important to clarify how the knowledge of the SIT could be of interest for the assessment of the vulnerability. Several reasons seem important to us:

- as the SIT value is based on measured data and does not require any supplementary hypotheses associated with the post elastic behaviour, the uncertainty is minimized,

- the fact that the SIT value is associated with normalized elastic response spectra should mean a real benefit for earthquake engineering practitioners. Moreover, site effects could be easily integrated by using specific spectra suited to the site,

- the comparison of the SIT value with the level of acceleration required by the seismic code gives an assessment of the potential of ductility that the structure should be able to develop; this can be a useful tool to identify the more critical cases, or to define a strategy of reinforcement,

- for the large number of buildings made of materials of low (slightly reinforced concrete) or very low (masonry) ductility, the SIT value can be a close indicator of the acceleration level leading to severe damages (or even collapse),

- finally, for specific buildings that should be kept in service, the SIT value should help to estimate whether the damage level remains acceptable.

\subsection{Case study on building $C$}

As an example, the SIT value is determined for the building $\mathrm{C}$ in the longitudinal direction. Firstly, the calculation of the strain limits of the structural elements - i.e. the transverse walls, the longitudinal lift shaft walls and the full precast panels (see Figure 4) - is presented. Then, the SIT results and comments will be addressed.

3.2.1. Internal strain limits of the structural elements. As presented in Section 2.2, the longitudinal building behaviour can be modelled by a pure shear beam. At a given floor level $i$, 

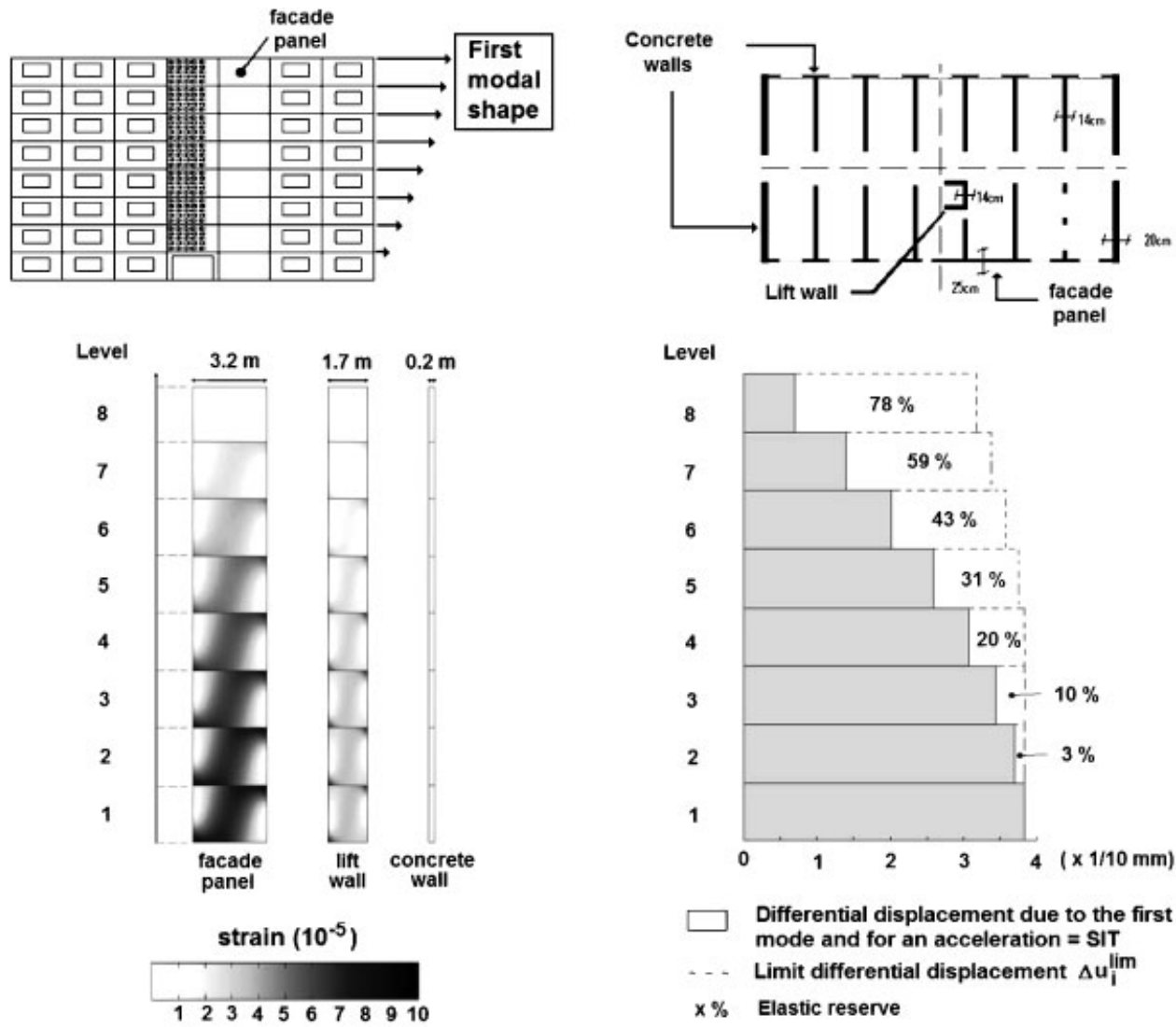

Figure 4. Strain limits of the main structural elements of the building C.

in each structural element $e$, the strain tensor $[\varepsilon]_{i, e}$ (non-uniform) results from:

(i) the strains $\left[\varepsilon^{d}\right]_{i, e}$ given by the dead loads (including the own load and for the carrier elements, the load of higher levels),

(ii) the strains $[\varepsilon]_{e}\left(\Delta u_{i}\right)$ imposed by the inter-storey displacement $\Delta u_{i}$ and composed by the shear contribution $\left[\varepsilon^{s}\right]_{e}\left(\Delta u_{i}\right)$ and the bending contribution $\left[\varepsilon^{b}\right]_{e}\left(\Delta u_{i}\right)$

$$
[\varepsilon]_{i, e}=\left[\varepsilon^{d}\right]_{i, e}+\left[\varepsilon^{s}+\varepsilon^{b}\right]_{e}\left(\Delta u_{i}\right)
$$

The strain tensor $\left[\varepsilon^{d}\right]_{i, e}$, is fully identified assuming a normal and uniform stress distribution in the horizontal cross-section (a modulus $E$ of $30 \mathrm{GPa}$ as experimentally determined and Poisson ratio $v$ of 0.2 is assumed). Using classical Strength of Material Theory, the strain tensors $\left[\varepsilon^{s}\right]$ and $\left[\varepsilon^{b}\right]$ are derived imposing a unit inter-storey displacement. This procedure, after diagonalization of $[\varepsilon]_{i, e}$, leads to an analytic expression (not detailed here) of the maximum tensile strain parameterized by inter-storey displacement $\Delta u_{i}$.

By imposing a limit tensile strain of $10^{-4}$, it is then possible to obtain, for each floor level $i$ and for any structural element $e$, the corresponding value of the relative displacement 
Table III. Maximum inter-storey displacements for important structural elements of building C.

\begin{tabular}{|c|c|c|c|c|c|c|c|c|}
\hline \multirow[b]{2}{*}{ Element } & \multicolumn{8}{|c|}{ Maximum inter-storey displacements $(1 / 10 \mathrm{~mm})$} \\
\hline & Level 1 & Level 2 & Level 3 & Level 4 & Level 5 & Level 6 & Level 7 & Level 8 \\
\hline \multicolumn{9}{|l|}{ Carrier elements } \\
\hline Transversal wall & 17.8 & 17.6 & 16.7 & 15.6 & 15.2 & 14.4 & 13.6 & 12.8 \\
\hline Lift wall & 4.4 & 4.4 & 4.2 & 4.0 & 3.8 & 3.6 & 3.4 & 3.2 \\
\hline \multicolumn{9}{|l|}{ Non-carrier elements } \\
\hline Full front panel & \multicolumn{4}{|c|}{$\underline{3.8}$} & \multicolumn{4}{|c|}{3.8} \\
\hline
\end{tabular}

$\Delta u_{i, e}^{\lim }$. Because of the variation of the compression force given by the dead load in carrier elements, it is expected to find different values at different floor levels. At the floor level $i$, the inter-storey displacement $\Delta u_{i}^{\lim }$ which triggers off the damage is the minimum of the $\Delta u_{i, e}^{\lim }$ values of all the elements of the storey.

For longitudinal vibrations of the building $\mathrm{C}$, the limit displacements for each floor level and for each type of structural element are presented in Table III. At the ground floor and the first three levels, it can be seen that the critical elements are the front precast panels. Above these three levels, the most critical elements become the walls of the lift cage.

3.2.2. Seismic integrity threshold of building $C$. Let us introduce $\left[U_{1}^{*}\right]$ and $\left[\Delta U_{1}^{*}\right]$, the normalized first mode eigenvector $\left(\left|U_{1}^{*}\right|=1\right)$ and the corresponding differential displacement vector, respectively. If $A$ is the amplitude of the first mode of vibration, then the displacement $\left[u_{1}\right]$ and differential displacement $\left[\Delta u_{1}\right]$ vectors are

$$
\left[u_{1}\right]=A *\left[U_{1}^{*}\right] \quad \text { and }\left[\Delta u_{1}\right]=A *\left[\Delta U_{1}^{*}\right]
$$

The amplitude $A_{i}$ that would trigger off the first structural damages at the floor level $i$, is calculated by writing

$$
\Delta u_{i}^{\lim }=A_{i} *\left[\Delta U_{1}^{*}\right]_{i}
$$

Finally, the amplitude $A_{\text {lim }}$ which triggers off the first damage in the whole building is the minimum of the $A_{i}$ values.

At this stage of the analysis, it remains to transform the value of $A_{\text {lim }}$ into an acceleration level. Conveniently, the seismic codes give the normalized elastic response spectra, i.e. the maximum response of a series of single-degree of freedom oscillators (SDOF) submitted to signals conform to the seismic spectra, with a reference acceleration of $a^{*}=1 \mathrm{~m} / \mathrm{s}^{2}$. According to the modal analysis, if $d^{*}\left(f_{1}\right)$ is the maximum SDOFs displacement response given by the normalized elastic response spectra at the 1 st mode frequency $f_{1}$, then the amplitude of modal response of the structure will be, for a standardized acceleration $S * a^{*}$

$$
A(S)=S * p_{1} * d^{*}\left(f_{1}\right)
$$

where $p_{1}={ }^{t}\left[U^{*}\right][M][1] /{ }^{t}\left[U^{*}\right][M]\left[U^{*}\right]$ is the first modal participation factor $\left(p_{1}=\pi / 4\right.$ for pure shear beam). The SIT is reached for a standardized acceleration SIT $* a^{*}$ such 
Table IV. SIT values for buildings $\mathrm{C}$ and $\mathrm{C}^{\prime}$-reference acceleration $a_{\text {ref }}$ for each zones: (zone $\mathrm{I}_{a}: 0.1 \mathrm{~g}$ ), (zone $\mathrm{I}_{b}: 0.15 \mathrm{~g}$ ), (zone II: $0.25 \mathrm{~g}$ ), (zone III: $0.35 \mathrm{~g}$ ).

\begin{tabular}{lcccc}
\hline Site condition & SIT $(\mathrm{g})$ & $\begin{array}{c}\text { Building C } \\
\text { Zone SIT }<a_{\text {ref }}\end{array}$ & SIT $(\mathrm{g})$ & $\begin{array}{c}\text { Building C' } \\
\text { Zone SIT }<a_{\text {ref }}\end{array}$ \\
\hline Good soil $S_{0}$ & 0.05 & $\mathrm{I}_{a} \rightarrow$ III & 0.09 & $\mathrm{I}_{a} \rightarrow$ III \\
Soft soil $S_{3}$ & 0.08 & $\mathrm{I}_{a} \rightarrow$ III & 0.18 & II $\rightarrow$ III \\
\hline
\end{tabular}

that $A(\mathrm{SIT})=A_{\lim }$, i.e.

$$
\mathrm{SIT}=\frac{A_{\lim }}{p_{1} * d^{*}\left(f_{1}\right)}
$$

Therefore, the SIT, i.e. the level of ground acceleration corresponding to seismic elastic limit of the building can be derived according to the code recommendations.

Considering a damping ratio of 5\%, the calculated values of the SIT for building $\mathrm{C}$ in the longitudinal direction are presented in Table IV. The calculation has been done considering that the structure is settled on the two extreme site conditions, $S_{0}$ (very good soil) and $S_{3}$ (soft soil) as defined by the French seismic code. Moreover, in order to investigate the sensibility of the SIT, the same calculations were developed for a fictitious building $\mathrm{C}^{\prime}$ identical to $\mathrm{C}$ but with a number of storey reduced to 4 (therefore more rigid with an higher first frequency).

3.2.3. Discussion. Table IV shows that, according to the site conditions, the SIT values of building $\mathrm{C}$ range between 0.05 and $0.08 \mathrm{~g}$. This order of magnitude is in agreement with the post-earthquake observations which showed that below $0.1 \mathrm{~g}$, there are very limited structural disorders in common concrete buildings. The SIT values of building $\mathrm{C}^{\prime}$, less loaded because of its higher frequency, are higher than for building $\mathrm{C}(0.09-0.18 \mathrm{~g})$.

If the SIT is lower than the acceleration required by the seismic code, it is believed that first damages would be induced by the reference earthquake. The French seismic code defines 4 zones, from zone $\left(\mathrm{I}_{b}\right)$ of weak seismicity to zone (III) of moderate seismicity and whose reference accelerations $a_{\text {ref }}$ are, respectively, equal to 0.1 and $0.35 \mathrm{~g}$. It seems that this particular structure $\mathrm{C}$ is quite vulnerable whatever the site and seismic zone are. As an aggravating factor it should be noted that the most critical structural element is the ground floor unreinforced panel for which a brittle failure can be expected.

The gap between the SIT and the reference acceleration of the seismic zone provides an indication of the ductility needed by the building to resist the reference earthquake. The larger is the gap between these accelerations, the more attention should be paid to the structure, and further investigations may be necessary to engage.

\subsection{Beyond the seismic integrity threshold?}

In practice, when analysing the post elastic behaviour, three different cases, depending on the ductility potential and its effective use during earthquake, can be distinguished:

- for buildings made of materials of low (slightly reinforced concrete) or very low (masonry) ductility, it can be considered that beyond the SIT the safety margin before severe 
damages is rather low; nevertheless the reserves of stability brought by hyperstatism may preserve from general collapse,

- for buildings made of ductile material (like confined reinforced concrete), additional information are needed to determine a plausible post-elastic scenario; two situations can be encountered: if the quasi-elastic mode shape clearly shows a level which concentrates the deformation (for instance in presence of a soft storey) the strength will essentially depend on the local ductility at this level, the ductility of other parts of the building remaining almost unemployed; if the design ends up in a regular mode shape, the ductility will be activated in the whole building, and the strength can be estimated using a push-over analysis [7] (assuming or knowing the amount and disposition of steel reinforcement).

\section{CONCLUSIONS}

This study shows the interest of the small amplitude auscultation methods in the seismic diagnosis of existing structures. Several experiments presented in Part I paper prove the robustness and the reliability of the information collected through ambient vibrations which enable the identification of the leading and negligible phenomena. Even if the data are limited to the quasi-elastic domain, their knowledge is of very first importance: the quasi-elastic behaviour is known to play an essential part especially for the dynamic amplification near eigenfrequencies, this phenomenon being one of the main reason of the structural damages.

It would have been possible to use sophisticated methods to obtain an accurate description of the buildings. However, keeping in mind the necessity of assessing a large number of buildings, simple modelling based on few elementary, though physical assumptions, are favoured. These approaches give an approximate description sufficiently realistic for engineering purposes.

In the same spirit, the concept of SIT, which is based on real data, presents practical advantages. It minimizes the use of uncertain assumptions on the non-linear post-elastic behaviour (at the three scales of the material, the structural elements and the structure) and it provides an acceleration level that can be easily compared with the reference accelerations given by the codes. From both reference accelerations and SIT values, the extent of the ductility needed by the structure can be evaluated. The confrontation of this value with the usual ductility of the present material could provide a good criterion to identify the most vulnerable structures. Although the results could be regarded as coherent, further developments should be done:

-For simplicity, the SIT concept was presented on the simple case of 'shear' building. Nevertheless, the same idea could be adapted for buildings where 'flexural' contribution is present (like building $\mathrm{G}$ ). In addition to the deformation related to the inter-storey drift, the deformation related to the inter-storey rotation has to be included. This latter induces a linear distribution of vertical motion, leading to extension or compression of the bearing elements. Except this modification, the procedure would remain similar.

- The SIT determination is rather easy for regular buildings. For irregular buildings, the beam modelling has to be adapted to include effects of torsion, soft storey, etc. An alternative approach would be to use an elastic numerical modelling fitted with the in situ data. 
- The simultaneous presence of several modes and the applications to buildings made of different materials could also be considered.

It should also be emphasized that, if the serviceability limits must be evaluated or a retrofitting needed, experimental data allow a good fitting of the numerical modelling. Indeed, whatever the complexity of the linear and non-linear numerical method, the results depend on the chosen assumptions. The knowledge of the quasi-elastic behaviour would help reduce the uncertainty of the modelling and define an adequate reinforcement owing to a better understanding of the structure.

As a conclusion, the association of experimental approach and simple models can be a good complement to the existing assessment methods of seismic vulnerability.

\section{APPENDIX A: MODAL ANALYSIS OF CLAMPED-FREE TIMOSHENKO CANTILEVER BEAMS}

The behaviour of a regular building of height $H$ made of elastic material (of Young's modulus $E$ ) is governed by the spatial distribution of columns, bearing walls and floors diaphragms constituting the storey (of height $h$ ). The linear mass of a storey is denoted $\Lambda$. In the framework of the modal analysis, the variables are expressed on the form $A(x, t)=A(x) \mathrm{e}^{\mathrm{i} \omega t}$, where $x$ is the position along the beam, $\omega$ the angular frequency and $t$ the time. For regular buildings, considering vibrations in a horizontal main direction, the deformations result from the two following contributions (see for instance Reference [6]):

(i) the bending motions characterized by the section rotation $\alpha$, the bending parameter EI and the associated momentum $M(x)=-\mathrm{EI} \alpha^{\prime}(x)$,

(ii) the shear motion characterized by the shear deflection $\beta$, the shear parameter denoted by $K$ and the associated shear force $T(x)=-K \beta(x)$.

The horizontal translation motion of the section, $U$, is related to the cinematical variables $\alpha, \beta$ by

$$
\alpha(x)=\beta(x)+U^{\prime}(x)
$$

Assuming the section rotation inertia $\mathrm{J} \omega^{2} \alpha(x)$ of the second-order (this hypothesis is verified a posteriori), the balance equations of a beam section are classically given by

$$
\begin{aligned}
T^{\prime}(x) & =-\Lambda \omega^{2} U(x) \\
M^{\prime}(x) & =T(x)
\end{aligned}
$$

These equations leads to a Timoshenko beam where translation motion of section, $U$, is governed by

$$
\mathrm{EI} U^{(4)}(x)+\frac{\mathrm{EI}}{K} \Lambda \omega^{2} U^{(2)}(x)=\Lambda \omega^{2} U(x)
$$

Introducing the wavelength $L=H / 2 \pi$ (i.e. the first modal wavelength of a clamped-free shear beam), the general harmonic solution of (A3) is in the form

$$
U(x)=a \cos \left(\delta_{1} \frac{x}{L}\right)+b \sin \left(\delta_{1} \frac{x}{L}\right)+c \cosh \left(\delta_{2} \frac{x}{L}\right)+d \sinh \left(\delta_{2} \frac{x}{L}\right)
$$


where $\left(i \delta_{1}\right)^{2}$ and $\delta_{2}^{2}$ are the two real roots of the equation

$$
\frac{\mathrm{EI}}{\Lambda \omega^{2} L^{4}} \delta^{4}+\frac{\mathrm{EI}}{K L^{2}} \delta^{2}-1=0
$$

The dimensionless parameter $C=\mathrm{EI} / K L^{2}$ characterizes the nature of the Timoshenko beam. This latter degenerates into an usual Euler-Bernoulli beam when $C=0$, and to a pure shear beam when $C=+\infty$.

The two dimensionless wave numbers $\delta_{1}$ and $\delta_{2}$ are related by

$$
\begin{aligned}
\delta_{1}^{2} \delta_{2}^{2} & =\frac{\Lambda \omega^{2} L^{4}}{\mathrm{EI}} \\
\delta_{1}^{2}-\delta_{2}^{2} & =\frac{\Lambda \omega^{2} L^{2}}{K}
\end{aligned}
$$

and therefore

$$
\frac{\delta_{1}^{2}-\delta_{2}^{2}}{\delta_{1}^{2} \delta_{2}^{2}}=C, \quad \delta_{2}=\frac{\delta_{1}}{\sqrt{1+C \delta_{1}^{2}}}
$$

The condition of existence of non-trivial solutions respecting the clamped-free boundary conditions, i.e. at the base $U(0)=0, \alpha(0)=0$, and at the top, $M(H)=0, T(H)=0$, leads to the following wave number equation involving the unique parameter $C$ :

$$
2\left(1+\cos \left(\delta_{1} \frac{\pi}{2}\right) \cosh \left(\delta_{2} \frac{\pi}{2}\right)\right)-C \sin \left(\delta_{1} \frac{\pi}{2}\right) \sinh \left(\delta_{2} \frac{\pi}{2}\right)+C^{2} \cos \left(\delta_{1} \frac{\pi}{2}\right) \cosh \left(\delta_{2} \frac{\pi}{2}\right)=0
$$

The infinite discrete series of roots $\delta_{1}$ of (A8) can be determined numerically. From the properties of trigonometric and hyperbolic functions, whatever the value of $C$, the series of roots $\delta_{1}$ are close to the odd integers sequel $2 k+1$ (indeed odd integers are exact solutions for pure shear beam $(C=+\infty)$ and, in other cases, provide excellent approximations from the third mode). Finally the eigenfrequencies are directly derived from (A6)

$$
f_{k}=\frac{1}{2 \pi L} \frac{\delta_{1 k}^{2}}{\sqrt{\left(\Lambda L^{2} / \mathrm{EI}\right)+\delta_{1 k}^{2} \Lambda / K}} \quad \text { with } \delta_{1 k} \simeq 2 k+1 ;(k \geqslant 2)
$$

\section{ACKNOWLEDGEMENTS}

This research was supported by the French Ministry of Environment and the French Association of Earthquake Engineering (AFPS).

\section{REFERENCES}

1. Benedetti D, Benzoni G, Parisi MA. Seismic vulnerability and risk evaluation for old urban nuclei. Earthquake Engineering and Structural Dynamics 1988; 16:183-201.

2. Fritz de la Orta GO. Screening methodology for the seismic assessment of particular buildings in Los Alamos national laboratory. 11th World Conference of Earthquake Engineering, Mexico, 1996. 
3. Hans S, Boutin C, Ibraim E, Roussillon P. In situ experiments and seismic analysis of existing buildings, Part I: Experimental investigations. Earthquake Engineering and Structural Dynamics 2005; 34.

4. Hans S. Auscultation des batiments existants. Ph.D. Thesis, ENTPE-INSA, Lyon, 2002.

5. Stephen NG. On the vibration of one-dimensional periodic structures. Journal of Sound and Vibration 1999; 227(5):1133-1142.

6. Boutin C, Hans S. Dynamics of periodic framed structures - continuum modelling by homogenisation. Computer and Geotechnics, Special Number: Homogenisation in Geomechanics 2003; 30(4):303-320.

7. Priestley MJN. Displacement-based seismic assessment of reinforced concrete buildings. Journal of Earthquake Engineering 1997; 1(1):157-192.

8. Gulkan P, Sozen MA, Demir S, Ersoy U. An alternative evaluation for determining seismic vulnerability of building structures. Acts of the Eleventh World Conference on Earthquake Engineering, Acapulco, Mexico, 22-27 June 1996. 